Annals of Pure and Applied Mathematics

Vol. 18, No. 1, 2018, 83-90

ISSN: 2279-087X (P), 2279-0888(online)

Published on 23 August 2018

Annals of

www.researchmathsci.org

DOI: http://dx.doi.org/10.22457/apam.v18n1a12

Pure and Applied

Mathematics

\title{
Interval Valued Q-fuzzy Quasi-ideals in a Semigroups
}

\author{
T.Selvakumar ${ }^{1}$ and M.V.Suresh ${ }^{2}$ \\ ${ }^{1}$ Department of Mathematics \\ ${ }^{1}$ St. Peter's University, Avadi, Chennai - 54, Tamilnadu, India \\ Email: tselvak93@gmail.com \\ ${ }^{2}$ Department of Mathematics \\ ${ }^{2}$ St. Peter's University, Avadi, Chennai - 54, Tamilnadu, India \\ Email: m.v.sureshpari@gmail.com \\ ${ }^{1}$ Corresponding author
}

Received 27 June 2018; accepted 14 August 2018

Abstract. We initiate the study of interval-valued Q-fuzzy quasi-ideal of a semigroup. In Section 2, we list some basic definitions in the later sections. In Section 3, we investigate interval-valued Q-fuzzy subsemigroups and in Section 4, we define interval valued Q-fuzzy quasi-ideals and establish some of their basic properties.

Keywords: interval-valued Q-fuzzy set, interval-valued Q- fuzzy left(right) ideal, interval-valued Q-fuzzy bi-ideal, interval-valued Q-fuzzy quasi-ideal.

AMS Mathematics Subject Classification(2010): 03E99, 20M99

\section{Introduction}

The theory of fuzzy sets proposed by Zadeh [13] in 1965 has achieved a great success in various fields. Since then, Ahsan and Latif [1] investigated fuzzy quasi-ideals in a semigroup. With the research of fuzzy sets, in 1965, Zadeh [14] introduced the notion of interval-valued fuzzy sets as a generalization of fuzzy sets. After then, Biswas [3] applied it to group theory. Rosenfeld [9] defined fuzzy subgroup and gave some of its properties. Rosenfeld's definition of fuzzy group is a turning point for pure Mathematicians. Since then, the study of fuzzy algebraic structures have been carried out in many directions such as semi-group, groups, rings, near-rings, modules, vector spaces, topology and so on.

Recently, Kang and Hur [4] studied interval-valued fuzzy subgroups and investigated some of its properties. Narayanan and Manikandan [8] studied Interval-valued fuzzy ideals generated by an interval-valued fuzzy subset in semi-groups and investigated some of its properties.

Thillaigovindan and Chinnadurai [11] studied on interval-valued fuzzy quasi-ideals of semi-groups and investigated some of its properties. Solairaju and Nagarajan [10] defined a new structure and constructions of Q-fuzzy group. Kim et al. [5] studied interval-valued fuzzy Quasi-ideals in a semigroups and investigated some of its properties. Murugadas et al. [7] studied interval-valued Q-fuzzy ideals generated by an interval-valued Q-fuzzy subset in ordered semi-groups and investigated some of its properties. Venkatesan and Sriram [12] studied multiplicative operations of IFMs of two operators namely $X_{1}$ and $X_{2}$ 
T.Selvakumar and M.V.Suresh

and investigated its algebraic properties.

In this paper, we initiate the study of interval-valued Q-fuzzy quasi-ideal of a semigroup. In Section 2, we list some basic definitions in the later sections. In Section 3, we investigate interval-valued Q-fuzzy subsemigroups and in Section 4, we define interval-valued Q-fuzzy quasi-ideals and establish some of their basic properties.

\section{Preliminaries}

In this section, we give to some basic definitions of interval-valued fuzzy set that are necessary for this paper.

Definition 2.1. Let $A, B \in D(I)^{X}$ and let $\left\{A_{\alpha}\right\}_{\alpha \in \Gamma} \subset D(I)^{X}$. Then

i) $A \subset B$ iff $A^{L} \leq B^{L}$ and $A^{U} \leq B^{U}$.

ii) $A=B$ iff $A \subset B$ and $B \subset A$.

iii) $A^{L}=\left[1-A^{U}, 1-A^{L}\right]$.

iv) $A \cup B=\left[A^{L} \vee B^{L}, A^{U} \vee B^{U}\right]$.

v) $A \cap B=\left[A^{L} \wedge B^{L}, A^{U} \wedge B^{U}\right]$.

vi) $\bigcup_{\alpha \in \Gamma} A_{\alpha}=\left[\mathrm{V}_{\alpha \in \Gamma} A_{\alpha}{ }^{L}, \mathrm{~V}_{\alpha \in \Gamma} A_{\alpha}{ }^{U}\right]$

vii) $\bigcap_{\alpha \in \Gamma} A_{\alpha}=\left[\bigwedge_{\alpha \in \Gamma} A_{\alpha}{ }^{L}, \bigwedge_{\alpha \in \Gamma} A_{\alpha}{ }^{U}\right]$.

Definition 2.2. Let $S$ be semigroup and let $\tilde{0} \neq A \in D(I)^{S}$. Then $A$ is called an:

i) Interval-valued Q-fuzzy semigroup of $S$ if $A^{L}(x y, q) \geq A^{L}(x, q) \wedge A^{L}(y, q)$ and $A^{U}(x y, q) \geq A^{U}(x, q) \wedge A^{U}(y, q)$ for any $x, y \in S, q \in Q$.

ii) Interval-valued Q-fuzzy left ideal of $S$ if $A^{L}(x y, q) \geq A^{L}(y, q)$ and $A^{U}(x y, q) \geq A^{U}(y . q)$ for any $x, y \in S, q \in Q$.

iii) Interval-valued Q-fuzzy right ideal of $S$ if $A^{L}(x y, q) \geq A^{L}(x, q)$ and $A^{U}(x y, q) \geq A^{U}(x, q)$ for any $x, y \in S, q \in Q$.

iv) Interval-valued Q-fuzzy (two sided) ideal of $S$ if it is both an IVLI and IVRI of $S$. We will denote the set of all IVSGs of $S$ as $\operatorname{IVSG}(S)$.

It is clear that $A \in \operatorname{IVI}(S)$ if and only if $A^{L}(x y, q) \geq A^{L}(x, q) \wedge A^{L}(y, q)$ and $A^{U}(x y, q) \geq A^{U}(x, q) \wedge A^{U}(y, q)$ for any $x, y \in S, q \in Q$ and if $A \in \operatorname{IVLI}(S)$, then $A \in \operatorname{IVSG}(S)$.

\section{Interval-valued Q-fuzzy subsemigroups}

In this section, we investigate interval-valued Q-fuzzy subsemigroups and its some algebraic properties.

Definition 3.1. A mapping $A=X \times Q \rightarrow D(I)$ is called an interval-valued Q-fuzzy set in $X$, denoted by $A=A^{L} \rightarrow A^{U}$ if $A^{L}, A^{U} \in I^{X}$ such that $A^{L} \leq A^{U}$, i.e., $A^{L}(x) \leq$ $A^{U}(x)$ for each $x \in X$ where $A^{L}(x, q)$ [respectively $A^{U}(x, q)$ ] is called the lower [respectively upper] end point of $x$ to $A$. For any $[a, b] \in D(I)$, the interval-valued fuzzy set $A$ in $X$ defined by $A(x)=\left[A^{L}(x, q), A^{U}(x, q)\right]=[a, b]$ for each $x \in X$ and $q \in Q$ is denoted by $[\widetilde{a, b}]$ and if $a=b$, then the IVS $[\widetilde{a, b}]$ is denoted by simply $\tilde{a}$. In particular, $\tilde{0}$ and $\tilde{1}$ denote the interval-valued Q-fuzzy empty set and the interval-valued Q-fuzzy whole set in X, respectively. We will denote the set of all IVSs in $X$ as $D(I)^{X}$. It is clear that set $A=\left[A^{L}, A^{U}\right] \in D(I)^{X}$ for each $A \in I^{X}$. 
Interval Valued Q-fuzzy Quasi-ideals in a Semigroups

Definition 3.2. Let $(X, \cdot)$ be a groupoid and let $A, B \in D(I)^{X}$. Then the interval-valued Q-fuzzy product of $A$ and $B$, denoted by $\left(A^{\circ} B\right)$, is an IVS in $X$ defined as follows :

For each $x \in X, q \in Q$

$$
(A \circ B)(x, q)=\left\{\begin{array}{lr}
{[a, b],} & \text { if } y z=x, q \in Q, \\
{[0,0],} & \text { otherwise. }
\end{array}\right.
$$

where $=\mathrm{V}_{y z=x}\left(A^{L}(y, q) \wedge B^{L}(z, q)\right), b=\mathrm{V}_{y z=x}\left(A^{U}(y, q) \wedge B^{U}(z, q)\right)$.

It is clear that for any $A, B, C \in D(I)^{X}$, if $B \subset C$, then $\left(A^{\circ} B\right) \subset\left(A^{\circ} C\right)$ and $\left(B{ }^{\circ} A\right) \subset\left(C{ }^{\circ} A\right)$.

Result 3.3. Let $(S$, .) be a groupoid.

i) If "." is associative, then so is " $\circ$ " in $D(I)^{S}$.

ii) If "." has an identity $e \in S$, then $e_{1} \in I V F_{P}(X)$ is an identity of ${ }^{\circ}$ in $D(I)^{S}$.

Proposition 3.4. Let $\mathrm{S}$ be a groupoid, let $Q$ be a non empty set and let $A, B, C \in D(I)^{S}$. Then

i) $A^{\circ}(B \cup C)=\left(A^{\circ} B\right) \cup\left(A^{\circ} C\right),(B \cup C){ }^{\circ} A=\left(B^{\circ} A\right) \cup\left(C^{\circ} A\right)$.

ii) $A^{\circ}(B \cap C) \subset\left(A^{\circ} B\right) \cap\left(A^{\circ} C\right),(B \cap C){ }^{\circ} A \subset\left(B{ }^{\circ} A\right) \cap\left(C^{\circ} A\right)$.

Proof: (i) Let $x \in S, q \in Q$ Suppose $x$ is not expressible as $x=y z$.

Then clearly $\left(A^{\circ}(B \cup C)\right)(x, q)=\tilde{0}=\left(\left(A^{\circ} B\right) \cup\left(A^{\circ} C\right)\right)(x, q)$.

Suppose $x$ is expressible as $x=y z$. Then

$\left(A^{\circ}(B \cup C)\right)(x, q)$

$$
\begin{aligned}
& =\bigvee_{x=y z}\left(A^{L}(y, q) \wedge(B \cup C)^{L}(z, q)\right) \\
& =\bigvee_{x=y z}\left(A^{L}(y, q) \wedge B^{L}(z, q)\right) \vee\left(A^{L}(y, q) \wedge C^{L}(z, q)\right) \\
& =\bigvee_{x=y z}\left(A^{L}(y, q) \wedge B^{L}(z, q)\right) \vee \bigvee_{x=y z}\left(A^{L}(y, q) \wedge C^{L}(z, q)\right) \\
& =\left(A^{\circ} B\right)^{L}(x, q) \vee\left(A^{\circ} C\right)^{L}(x, q) \\
& =\left(\left(A^{\circ} B\right) \cup\left(A^{\circ} C\right)\right)^{L}(x, q)
\end{aligned}
$$

Thus $A^{\circ}(B \cup C)=\left(A^{\circ} B\right) \cup\left(A^{\circ} C\right)$. By the similar arguments, we have $(B \cup C)^{\circ} A=\left(B^{\circ} A\right) \cup\left(C^{\circ} A\right)$.

ii) Let $x \in S, q \in Q$. Suppose $x$ is not expressible as $x=y z$.

Then clearly $\left(A^{\circ}(B \cup C)\right)(x, q)=\tilde{0}=\left(\left(A^{\circ} B\right) \cap\left(A^{\circ} C\right)\right)(x, q)$.

Suppose $x$ is expressible as $x=y z$. Then

$$
\begin{aligned}
\left(A^{\circ}(B \cap C)\right)(x, q) & =\bigvee_{x=y z}\left(A^{L}(y, q) \wedge(B \cap C)^{L}(z, q)\right) \\
& =\bigvee_{x=y z}\left(A^{L}(y, q) \wedge B^{L}(z, q)\right) \wedge\left(A^{L}(y, q) \wedge C^{L}(z, q)\right) \\
& =\bigvee_{x=y z}\left(A^{L}(y, q) \wedge B^{L}(z, q)\right) \wedge \bigvee_{x=y z}\left(A^{L}(y, q) \wedge C^{L}(z, q)\right) \\
& =\left(A^{\circ} B\right)^{L}(x, q) \wedge\left(A^{\circ} C\right)^{L}(x, q)
\end{aligned}
$$


T.Selvakumar and M.V.Suresh

$$
=\left(\left(A^{\circ} B\right) \cap\left(A^{\circ} C\right)\right)^{L}(x, q) \text {. }
$$

Similarly, we have that $\left(A^{\circ}(B \cap C)\right)^{U}(x, q) \leq\left(\left(A^{\circ} B\right) \cap\left(A^{\circ} C\right)\right)^{U}(x, q)$.

Thus $A^{\circ}(B \cap C) \subset\left(A^{\circ} B\right) \cap\left(A^{\circ} C\right)$.

By the similar arguments, we have $(B \cap C)^{\circ} A \subset\left(B^{\circ} A\right) \cap\left(C^{\circ} A\right)$.

Proposition 3.5. Let $S$ be a semigroup and let $\tilde{0} \neq A \in D(I)^{S}$. Then $A \in \operatorname{IVSG}(S)$ if and only if $\left(A^{\circ} A\right) \subset A$.

Result 3.6. Let $A$ be a non-empty subset of a semigroup $S$.

i) $A$ is a subsemigroup of $S$ if and only if $[\chi A, \chi A] \in \operatorname{IVSG}(\mathrm{S})$.

ii) $A \in \operatorname{LI}(S)$ if and only if $\left[\chi_{A}, \chi_{A}\right] \in \operatorname{IVLI}(S)$.

Result 3.7. Let $S$ be a semigroup and let $\tilde{0} \neq A \in D(I)^{S}$. Then $A \in \operatorname{IVLI}(S)$ if and only if $\left(\tilde{1}^{\circ} A\right) \subset A$.

Proposition 3.8. Let $S$ be a semigroup and let $A, B, C \in D(I)^{S}, q \in Q$. If $A \subset B$, then $\left(A^{\circ} C\right) \subset\left(B{ }^{\circ} C\right)$ and $\left(C^{\circ} A\right) \subset\left(C^{\circ} B\right)$.

Proof: Let $x \in S, q \in Q$. Suppose $(x, q)$ is not expressible as $(x, q)=(y z, q)$. Then clearly $\left(A^{\circ} C\right)(x, q)=\tilde{0}=\left(B^{\circ} C\right)(x, q)$. Suppose $x$ is not expressible as $x=y z$. Then

$$
\left(A^{\circ} C\right)^{L}(x, q)
$$

$$
\begin{aligned}
& =\bigvee_{x=y z}\left(A^{L}(y, q) \wedge C^{L}(z, q)\right) \\
& =\bigvee_{x=y z}\left(B^{L}(y, q) \wedge C^{L}(z, q)\right) \\
& =\left(B^{\circ} C\right)^{L}(x, q) .
\end{aligned}
$$

Similarly, we have that $\left(A^{\circ} C\right)^{U}(x, q) \leq\left(B^{\circ} C\right)^{U}(x, q)$.

Hence $\left(A^{\circ} C\right) \subset\left(B^{\circ} C\right)$. By the similar arguments, we have $\left(C^{\circ} A\right) \subset\left(C^{\circ} B\right)$.

\section{Interval valued Q-fuzzy quasi-ideals}

A nonempty subset $A$ of a semigroup $S$ is called a quasi- ideal of $S$ if $A S \cap S A \subset A$.

We will denote the set of all quasi-ideals of $S$ as QI(S).

Definition 4.1. Let $S$ be a semigroup and let $\tilde{0} \neq A \in D(I)^{S}$. Then $A$ is called an interval-valued fuzzy quasi-ideal (in short, $I V Q I)$ of $S$ if $\left(\tilde{1}^{\circ} A\right) \cap\left(A^{\circ} \tilde{1}\right) \subset A$.

We will denote the set of all IVQIs of $S$ as IVQI(S).

Example 4.2. Let $S=\{a, b, c\}$ be any semigroup with the following multiplication table:

We define a mapping $A: S \rightarrow D(I)$ as follows:

$A(a)=[0.1,0.8], A(b)=[0.1,0.8], A(c)=[0.3,0.6]$.

Then we can see that $A \in \operatorname{IVQI}(S)$.

Theorem 4.3. Let $A$ be a nonempty subset of a semigroup $S$. Then $A \in Q I(S)$ if and 
Interval Valued Q-fuzzy Quasi-ideals in a Semigroups

only if $\left[\chi_{A}, \chi_{A}\right] \in \operatorname{IVQI}(S)$.

\begin{tabular}{|c|c|c|c|}
\hline$\cdot$ & $a$ & $b$ & $c$ \\
\hline$a$ & $a$ & $a$ & $a$ \\
\hline$b$ & $a$ & $b$ & $b$ \\
\hline$c$ & $a$ & $a$ & $b$ \\
\hline
\end{tabular}

Proof: Suppose $A \in Q I(S)$ and let $x \in S$. Suppose $x \in A, q \in Q$. Then clearly $\chi_{A}(x, q)=1 \geq\left(\left(\tilde{1}^{\circ}\left[\chi_{A}, \chi_{A}\right]\right) \cap\left(\left[\chi_{A}, \chi_{A}\right]^{\circ} \tilde{1}\right)\right)^{L}(x, q)$.

Thus $\left(\tilde{1}^{\circ}\left[\chi_{A}, \chi_{A}\right]\right) \cap\left(\left[\chi_{A}, \chi_{A}\right]^{\circ} \tilde{1}\right) \subset\left[\chi_{A}, \chi_{A}\right]$. Suppose $x \notin A$. Then either $x$ is expressible as $x=y z$ or not.

Case (i): Suppose $x$ is not expressible as $x=y z$. Then

$$
\left(\left(\tilde{1}^{\circ}\left[\chi_{A}, \chi_{A}\right]\right) \cap\left(\left[\chi_{A}, \chi_{A}\right]^{\circ} \tilde{1}\right)\right)(x, q)=\tilde{1}=\left[\chi_{A}, \chi_{A}\right](x, q) .
$$

Case (ii): Suppose $x$ is expressible as $x=y z$.

Since $x \notin A$, either $y \in A$ or $z \notin A$. If $y \in A$ and $z \notin A$, then there cannot be another expression of the form $x=a b$, where $a \notin A$ and $b \in A$ (Assume that there exist $a \notin A$ and $b \in A$ such that $x=a b$. Then $x \in S A \cap A S \subset A$. Thus $x \in A$.

This contradicts the fact that $x \notin A)$. Thus either $\left(\tilde{1}^{\circ}\left[\chi_{A}, \chi_{A}\right]\right)(x, q)=\tilde{0}$ or $\left(\left[\chi_{A}, \chi_{A}\right]^{\circ} \tilde{1}\right)(x, q)=\tilde{0}$. So $\left(\left(\tilde{1}^{\circ}\left[\chi_{A}, \chi_{A}\right]\right) \cap\left(\left[\chi_{A}, \chi_{A}\right]^{\circ} \tilde{1}\right)\right)(x)=\tilde{0}$.

Then $\left(\left(\tilde{1}^{\circ}\left[\chi_{A}, \chi_{A}\right]\right) \cap\left(\left[\chi_{A}, \chi_{A}\right]^{\circ} \tilde{1}\right)\right) \subset\left[\chi_{A}, \chi_{A}\right]$. Hence, in all, $\left[\chi_{A}, \chi_{A}\right] \in \operatorname{IVQI}(S)$.

Conversely, suppose the necessary condition holds.

Let $x \in S A \cap A S$. Then $x \in S A$ and $x \in A S$. Thus there exist $a, a^{\prime} \in A$ and $s, s^{\prime} \in S$ such that $x=s a$ and $x=a^{\prime} s^{\prime}$. So

$$
\begin{aligned}
\left(\left(\tilde{1}^{\circ}\left[\chi_{A}, \chi_{A}\right]\right)\right. & \left.\cap\left(\left[\chi_{A}, \chi_{A}\right]^{\circ} \tilde{1}\right)\right)^{L}(x, q) \\
= & \left(\tilde{1}^{\circ}\left[\chi_{A}, \chi_{A}\right]\right)^{L}(x, q) \wedge\left(\left[\chi_{A}, \chi_{A}\right]^{\circ} \tilde{1}\right)^{L}(x, q) \\
& =\bigvee_{x=y z}\left(\tilde{1}^{L}(y, q) \wedge \chi_{A}(z, q)\right) \wedge \bigvee_{x=y z}\left(\chi_{A}{ }^{L}(y, q) \wedge \chi_{S}{ }^{L}(z, q)\right) \\
& \geq\left(\chi_{S}(s) \wedge \chi_{A}(a)\right) \wedge\left(\chi_{A}\left(a^{\prime}\right) \wedge \tilde{1}^{L}\left(s^{\prime}\right)\right) \\
& =1 .
\end{aligned}
$$

Similarly, we have that $\left(\left(\tilde{1}^{\circ}\left[\chi_{A}, \chi_{A}\right]\right) \cap\left(\left[\chi_{A}, \chi_{A}\right]^{\circ} \tilde{1}\right)\right)^{U}(x) \geq 1$. Then, by the hypothesis, $\chi_{A}(x) \geq 1$. Thus $x \in A$. So $S A \cap A S \subset A$. Hence $A \in Q I(S)$.

Definition 4.4. A nonempty fuzzy set $A$ of a semigroup $S$ is called a Q-fuzzy quasi-ideal of $S$ if $\left(\chi_{S}{ }^{\circ} A\right) \wedge\left(A^{\circ} \chi_{S}\right) \leq A$, where $\chi_{S}$ is the whole fuzzy set defined by $\chi_{S}(x, q)=1$ for each $x \in S, q \in Q$.

Remark 4.5. Let $S$ be a semigroup.

i) If $A$ is a Q-fuzzy quasi-ideal of $S$, then $[A, A] \in \operatorname{IVQI}(S)$.

ii) If $A \in \operatorname{IVQI}(S)$, then $A L$ and $A U$ are Q-fuzzy quasi-ideals of $S$. 
T.Selvakumar and M.V.Suresh

Proposition 4.6. Let $S$ be a semigroup. Then $\operatorname{IVQI}(S) \subset \operatorname{IVSG}(S)$.

Proof: Let $A \in I V Q I(S)$. Since $A \subset \tilde{1}$, by Proposition 3.8, $A^{\circ} A \subset \tilde{1}^{\circ} A$ and $A^{\circ} A \subset A^{\circ} \tilde{1}$. Then $A^{\circ} A \subset\left[\tilde{1}^{\circ} A\right] \cap\left[A^{\circ} \tilde{1}\right]$. Since $A \in I V Q I(S),\left(\tilde{1}^{\circ} A\right) \cap$ $\left(A^{\circ} \tilde{1}\right) \subset A$. Thus $A^{\circ} A \subset A$.

Hence, by Proposition 3.5, $A \in \operatorname{IVSG}(S)$.

Definition 4.7. Let $S$ be a semigroup and let $\dot{\tilde{0}} \neq A \in D(I)^{S}, q \in Q$.

Then $A$ is called an interval-valued Q-fuzzy bi-ideal (in short, $I V B I$ ) of $S$ if it satisfies the following conditions: for any $x, y, z \in S, q \in Q$.

i) $A^{L}(x y, q) \geq A^{L}(x, q) \wedge A^{L}(y, q)$ and $A^{U}(x y, q) \geq A^{U}(x, q) \wedge A^{U}(y, q)$.

ii) $A^{L}(x y z, q) \geq A^{L}(x, q) \wedge A^{L}(z, q)$ and $A^{U}(x y z, q) \geq A^{U}(x, q) \wedge A^{U}(z, q)$.

We will denote the set of all IVBIs of $S$ as IVBI(S).

Result 4.7. Let $A$ be a nonempty subset of a semigroup. Then $A \in B I(S)$ if and only if $\left[\chi_{A}, \chi_{A}\right] \in \operatorname{IVBI}(S)$.

Theorem 4.8. Let $S$ be a semigroup and let $\dot{\tilde{0}} \neq A \in D(I)^{S}, q \in Q$.

Then $A \in \operatorname{IVBI}(S)$ if and only if $A{ }^{\circ} A \subset A$ and $A \circ \tilde{1}^{\circ} A \subset A$.

Proof: Suppose $A \in I V B I(S)$. From Proposition 3.5, $A \circ A \subset A$. Let $x \in S, q \in Q$

Suppose $x$ is not expressible as $x=y z$. Then clearly $(A \circ \tilde{1} \circ A)(x, q)=\tilde{0}$.

Thus $A \circ \tilde{1} \circ A \subset A$.

Suppose $x$ is expressible as $x=y z$. Then $\left(A^{\circ} \tilde{1}^{\circ} A\right)(x, q) \neq \tilde{0}$. Thus $\left(A^{\circ} \tilde{1}^{\circ} A\right)^{L}(x, q)$

and

$$
=\bigvee_{x=y z}\left(A^{L}(y, q) \wedge\left(\tilde{1}^{\circ} A\right)^{L}(z, q)\right)>0
$$

$$
=\bigvee_{x=y z}\left(A^{U}(y, q) \wedge\left(\tilde{1}^{\circ} A\right)^{U}(z, q)\right)>0
$$

So $\left(\tilde{1}^{\circ} A\right)^{L}(z, q)>0$ and $\left(\tilde{1}^{\circ} A\right)^{U}(z, q)>0$. Then there exist $u, v \in S$ with $z=u v$ such that

$$
\begin{aligned}
& \left.\left(\tilde{1}^{\circ} A\right)^{L}(z, q) \quad=\bigvee_{z=u v}\left(\tilde{1}^{L}(u, q) \wedge A^{L}(q, q)\right)=\bigvee_{z=u v} A^{L}(v, q)\right) \\
& \text { and } \\
& \left.=\bigvee_{z=u v}\left(\tilde{1}^{U}(u, q) \wedge A^{U}(v, q)\right)=\bigvee_{z=u v} A^{U}(v, q)\right)
\end{aligned}
$$

Since $A \in \operatorname{IVBI}(S), A^{L}(x, q)=A^{L}(y u v, q) \geq A^{L}(y, q) \wedge A^{L}(v, q)$ and $A^{U}(x, q)=A^{U}(y u v, q) \geq A^{U}(y, q) \wedge A^{U}(v, q)$. Then $A^{L}(x, q)$

and

$$
\geq \bigvee_{x=y z}\left(A^{L}(y, q) \wedge\left(\bigvee_{z=u v} A^{L}(q, q)\right)\right)=\left(A^{\circ} \tilde{1}^{\circ} A\right)^{L}(x)
$$


Interval Valued Q-fuzzy Quasi-ideals in a Semigroups

$\geq \bigvee_{x=y z}\left(A^{U}(y, q) \wedge\left(\bigvee_{z=u v} A^{U}(q, q)\right)\right)=\left(A^{\circ} \tilde{1}^{\circ} A\right)^{U}(x)$

Hence, in all, $A^{\circ} \tilde{1}^{\circ} A \subset A$.

Conversely, suppose the necessary condition holds.

Since $A \circ A \subset A$, it is clear that the following hold:

$A^{L}(x y, q) \geq A^{L}(x, q) \wedge A^{L}(y, q)$ and $A^{U}(x y, q) \geq A^{U}(x, q) \wedge A^{U}(y, q)$.

For any $x, y \in S, q \in Q$. Let $x, y, z \in S$ and let $u=x y z$. Then

$A^{L}(x y z)=A^{L}(p)$

$$
\begin{aligned}
& \geq\left(A \circ \tilde{1}^{\circ} A\right)^{L}(u) \\
& =\bigvee_{u=s t}\left(A^{L}(s, q) \wedge\left(\tilde{1}^{\circ} A\right)^{L}(t, q)\right) \\
& \geq A^{L}(x, q) \wedge\left(\tilde{1}^{\circ} A\right)^{L}(y z, q) \\
& =A^{L}(x, q) \wedge \vee_{y z=a b}\left(\tilde{1}^{L}(a, q) \wedge A^{L}(b, q)\right) \\
& \geq A^{L}(x, q) \wedge A^{L}(y, q) \wedge A^{L}(z, q) \\
& =A^{L}(x, q) \wedge A^{L}(z, q) .
\end{aligned}
$$

Similarly, we have that $A^{U}(x y z, q) \geq A^{U}(x, q) \wedge A^{U}(z, q)$.

Hence, $A \in \operatorname{IVBI}(S)$.

Proposition 4.9. Let $S$ be a semigroup. Then $\operatorname{IV} Q I(S) \subset I V B I(S), q \in Q$.

Proof: Let $A \in \operatorname{IVQI}(S)$. Then, by Proposition 4.6, $A \in \operatorname{IVSG}(S)$.

Thus $A^{L}(x y, q) \geq A^{L}(x, q) \wedge A^{L}(y, q)$ and $A^{L}(x y, q) \geq A^{L}(x, q) \wedge A^{L}(y, q)$ for any $x, y \in S, q \in Q$.

So, by Proposition 3.5, $A \circ A \subset A$. It is clear that $A \circ \tilde{1} \subset \tilde{1}$ and $\tilde{1} \circ A \subset \tilde{1}$.

Then, by Proposition 3.8, $A \circ \tilde{1} \circ A \subset \tilde{1} \circ A$ and $A \circ \tilde{1} \circ A \subset A \circ \tilde{1}$.

Thus $A^{\circ} \tilde{1}^{\circ} A \subset[\tilde{1} \circ A] \cap\left[A^{\circ} \tilde{1}\right] \subset A$.

Hence, by Theorem 4.8, $A \in \operatorname{IVBI}(S)$.

\section{Conclusions}

In this paper, we initiate the study of interval-valued fuzzy quasi-ideal of a semigroup and investigate interval-valued Q-fuzzy subsemigroups and define interval-valued Q-fuzzy quasi-ideals and establish some of their basic properties.

\section{REFERENCES}

1. J.Ahsan and R.M.Latif, Fuzzy quasi-ideals in semigroups, J. Fuzzy Math., 9 (2) (2001) 259-270.

2. R. Biswas, Rosenfeld's fuzzy subgroups with interval-valued membership functions, Fuzzy Sets and Systems, 63 (1995) 87-90.

3. A.H.Clifford and G.B.Preston, The Algebraic Theory of Semigroup 1, AMS, Soc. Providence, 1961.

4. H.W.Kang and K.Hur, Interval-valued fuzzy subgroups and subrings, Honam Math. J, 32 (4) (2010) 543-617.

5. S.-M.Kim, K.Hur, M.Cheng and G.-B.Chae, Interval valued fuzzy quasi-ideals in a semigrups, Internatinal journal of Fuzzy Logic and Intelligent Systems, 12(3) (2012) 215-225. 
T.Selvakumar and M.V.Suresh

6. T.K.Mondal and S. K. Samanta, Topology of interval-valued fuzzy sets, Indian J. Pure Appl. Math., 30 (1) (1999) 20-38.

7. P.Murugadas, A.Arikrishnan and M.R.Thirumagal, Interval-valued q-fuzzy ideals generated by an interval-valued q-fuzzy subset in ordered semi-groups, Annals of Pure and Applied Mathematics, 13(2) (2017) 211-222.

8. A.L.Narayanan and T.Manikandan, Interval-valued fuzzy ideals generated by an interval-valued fuzzy subset in semi-groups, J.App. Maths and Computing, 20 (2006) 455-465.

9. A.Rosenfeld, Fuzzy groups, Journal of Mathematical Analysis and Applications, 35 (1971) 512-517.

10. A.Solairaju and R.Nagarajan, A new structure and constructions of Q-fuzzy group, Advance in Fuzzy Mathematics, 4 (2009) 23-29.

11. N.Thillaigovindan and V.Chinnadurai, On interval-valued fuzzy quasi-ideals of semi-groups, East Asian Mathematical Journal, 25(4) (2009) 441-453.

12. D. Venkatesan and S. Sriram, Multiplicative operations of intuitionist fuzzy matrices, Annals of Pure and Applied Mathematics,14(1) (2017) 173-181.

13. L.A.Zadeh, Fuzzy sets, Inform. and Centrol., 8 (1965) 338-353.

14. L.A.Zadeh, The concept of a linguistic variable and its application to approximate reasoning I, Inform. Sci., 8 (1975) 199-249. 